\title{
Generation of initial molecular dynamics configurations in arbitrary geometries and in parallel
}

\author{
Graham B. Macpherson*, Matthew K. Borg and Jason M. Reese \\ Department of Mechanical Engineering, University of Strathclyde, \\ Glasgow G1 1XJ, UK \\ (draft September 19, 2007)
}

\begin{abstract}
A computational pre-processing tool for generating initial configurations of molecules for molecular dynamics simulations in geometries described by a mesh of unstructured arbitrary polyhedra is described. The mesh is divided into separate zones and each can be filled with a single crystal lattice of atoms. Each zone is filled by creating an expanding cube of crystal unit cells, initiated from an anchor point for the lattice. Each unit cell places the appropriate atoms for the user-specified crystal structure and orientation. The cube expands until the entire zone is filled with the lattice; zones with concave and disconnected volumes may be filled. When the mesh is spatially decomposed into portions for distributed parallel processing, each portion may be filled independently, meaning that the entire molecular system never needs to fit onto a single processor, allowing very large systems to be created. The computational time required to fill a zone with molecules scales linearly with the number of cells in the zone for a fixed number of molecules, and better than linearly with the number of molecules for a fixed number of mesh cells. Our tool, molConfig, has been implemented in the open source C++ code OpenFOAM.
\end{abstract}

Keywords: molecular dynamics, nano fluidics, molecular geometry definition, parallel computing, OpenFOAM PACS: 31.15.Qg, 47.11.Mn

\section{Introduction and motivation}

Molecular Dynamics (MD) simulations in arbitrary geometries defined by a mesh comprising unstructured, arbitrary polyhedral cells, require initial configurations of molecules to be generated corresponding to the volumes defined by the mesh. This paper describes the algorithms underpinning a pre-processing tool able to create such configurations: molConfig. It is able to fill volumes defined by a zone of the mesh (a set of cells) with a single species crystal lattice of molecules. The user may specify the lattice structure, orientation, density, temperature and average velocity.

To preserve generality in the algorithm description, 'molecule' will be used in this paper to mean 'atom', 'ion' or 'molecule', i.e. a region being filled with argon atoms, with sodium and chloride ions, or with whole water molecules would all be referred to as being filled with molecules. The physical context should indicate the meaning.

molConfig is written using OpenFOAM [1], an open source $\mathrm{C}++$ toolbox. It provides molecule configuration generation for an MD code, also written using OpenFOAM, that performs simulations in meshes of unstructured arbitrary polyhedra [2]. This type of mesh allows complex, realistic engineering geometries derived from CAD models to be directly simulated by MD; it is intended for the simulation and design of nano-scale devices. molConfig operates independently on individual portions of a mesh that has been spatially decomposed to run in parallel, allowing systems comprising very large numbers of molecules to be created because they never need to all be contained on a single processor. The molecular configurations are the same whether generated in parallel or in serial; crystal lattices generated in parallel are continuous and defectless across interprocessor boundaries. All parallel decomposition, interprocessor communication (using MPI), and reconstruction is dealt with by OpenFOAM.

*Corresponding author: graham.macpherson@strath.ac.uk 


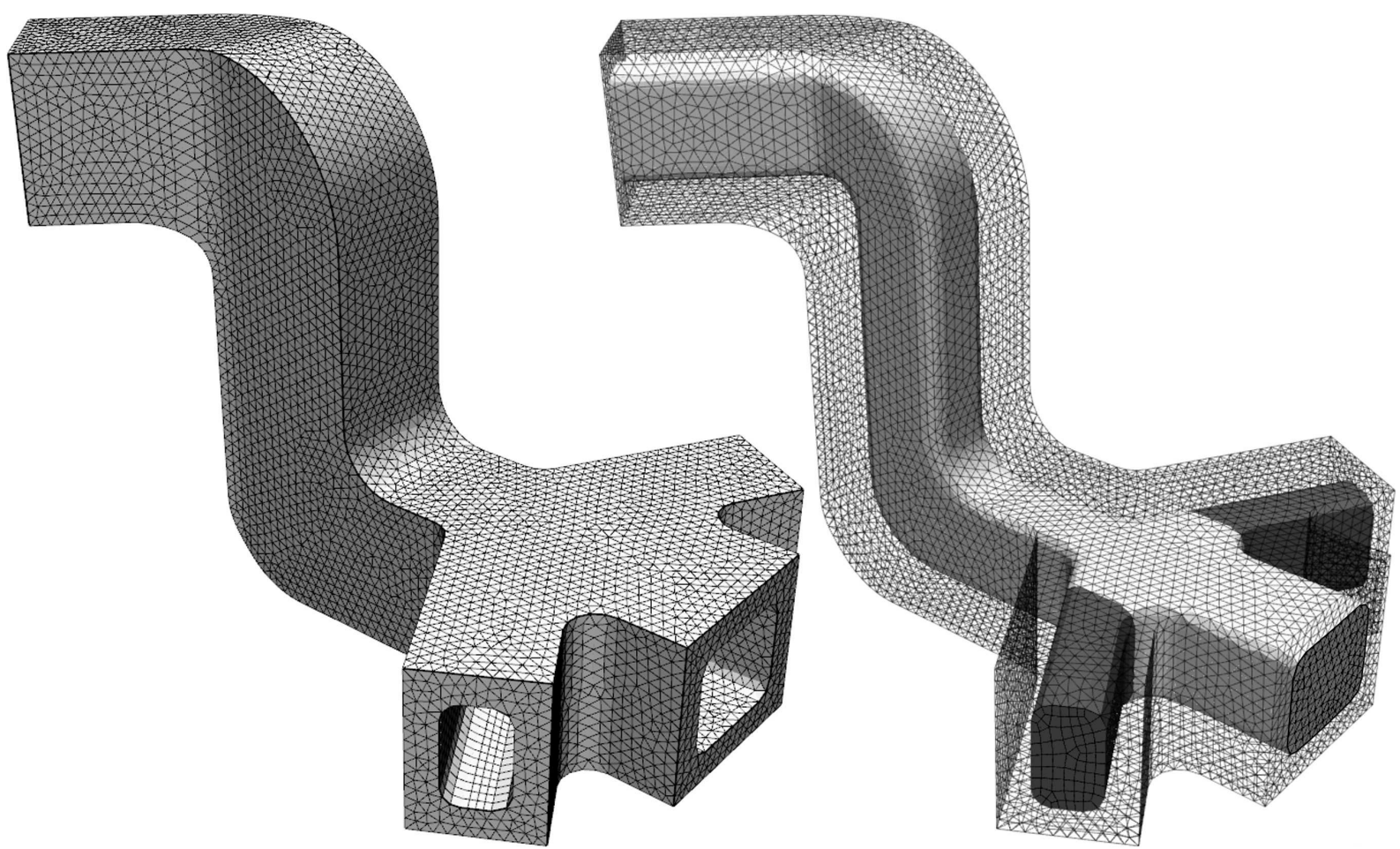

Figure 1. A complex geometry test case: a three-inlet fluid mixing channel. Four zones are defined, the exterior region (left) contains a tethered crystal lattice to define the channel walls. The three interior zones (right, one central and two 'arm' zones) contain the molecules representing the liquid. The overall height of the system is $82 \mathrm{~nm}$.

\subsection{Mesh description}

The mesh in OpenFOAM is flexible and powerful: it is unstructured and built from arbitrary polyhedra. From the OpenFOAM user guide [1]:

'By default OpenFOAM defines a mesh of arbitrary polyhedral cells in 3D, bounded by arbitrary polygonal faces, i.e. the cells can have an unlimited number of faces where, for each face, there is no limit on the number of edges nor any restriction on its alignment.'

A list of mesh vertex positions is stored and a list of mesh faces is constructed; each face is an ordered list of vertex numbers. Cells are constructed as a list of face numbers. Cells can be grouped together into zones, each zone representing a region of the domain with common characteristics. Zones are used in molConfig to define regions to be filled with different crystals.

\subsection{Motivation: example application}

The motivation for developing a molecular configuration generator for arbitrary geometries is the desire to use MD to simulate systems such as that shown in figure 1 . This example case is a three-inlet mixing chan-

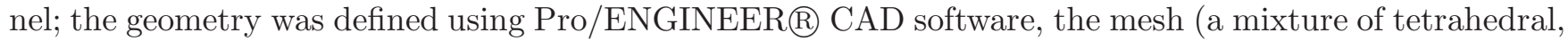
hexahedral and pyramidal cells) was generated using GAMBITß(a commercial mesh generator) then the mesh was imported into OpenFOAM. Four zones of cells were defined and the mesh was decomposed into 52 portions for parallel processing (see figure 2) each of which was filled with molecules independently.

\section{Volume filling algorithm}

The algorithm used by molConfig fills any volume, defined by a zone of cells, with a single lattice of molecules with specified orientation and alignment. It generates an expanding cube of lattice unit cells, starting from a user-specified position (known as an 'anchor' for the lattice), and angled according to a user-specified orientation (see figure 3). Each of these unit cells is populated with molecules corresponding 


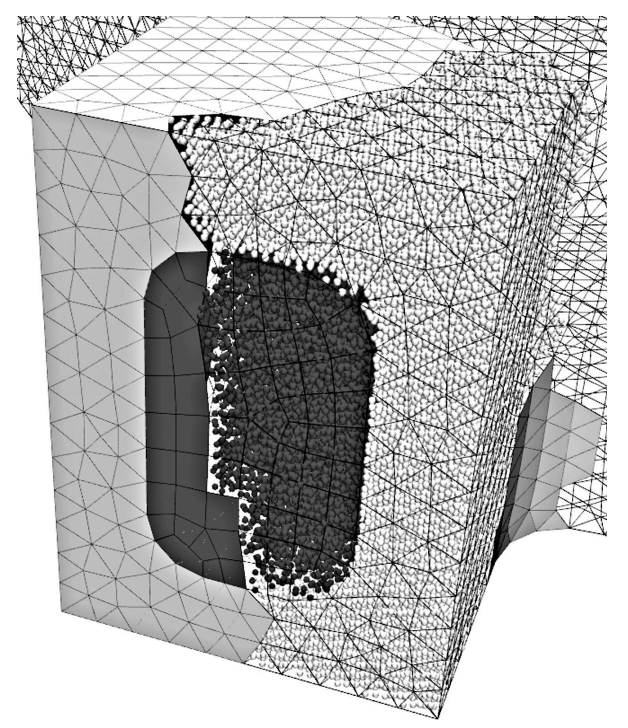

Figure 2. The test geometry (see figure 1) was decomposed into 52 portions for parallel processing — the portions were filled with molecules in parallel. Approximately 48000 molecules contained in one portion are shown here. Two different types of molecule are shown: wall molecules (light) tethered in an FCC crystal and liquid molecules (dark) which are free to move. The four zones were filled with a total of approximately 2.2 million molecules.

to a user-specified type of lattice for the zone. Each proposed molecule position is tested to see which cell it occupies ${ }^{1}$. If this cell comprises part of the zone currently being filled, the molecule is accepted; if not, the molecule is rejected. Using lattice unit cells makes the generation of the expanding cube independent of the specific crystal structure. Additional layers of unit cells are added to the cube until no molecules are accepted for placement in a complete layer, meaning that the volume has been completely filled. An exception is made if no molecules have been placed in the zone so far, enabling the lattice anchors to be placed outside the volume of the zone.

Concave and disconnected volumes of cells belonging to the same zone must be able to be filled (see figure 3) which makes more sophisticated algorithms (for example projecting rays to find the extents of the domain) difficult to implement reliably. The ability to fill disconnected volumes allows, for example, wall regions with gaps for fluid inlets to be filled with a single lattice. It is also a requirement for parallelisation because mesh decomposition will often produce disconnected volumes for a zone that is fully-connected in the undecomposed mesh.

\subsection{Creating an expanding cube of unit cells}

Consider a solid cube of unit cells comprising $n$ layers of concentric hollow cubes of one unit cell thickness, starting with $n=0$ as a single unit cell at the centre. A layer of this expanding cube is constructed as a combination of a top and bottom 'cap' plus 'rings' of cells, see figure 4. A cartesian lattice coordinate system local to the expanding cube is defined to specify the position of the centre of a unit cell,

$$
\boldsymbol{\Lambda}=\Lambda_{x} \mathbf{x}_{\lambda}+\Lambda_{y} \mathbf{y}_{\lambda}+\Lambda_{z} \mathbf{z}_{\lambda} \equiv\left(\Lambda_{x}, \Lambda_{y}, \Lambda_{z}\right)
$$

where $\Lambda_{x}, \Lambda_{y}$ and $\Lambda_{z}$ are integers for unit cell centres and $\mathbf{x}_{\lambda}, \mathbf{y}_{\lambda}$ and $\mathbf{z}_{\lambda}$ are the lattice coordinate system unit vectors.

Caps. Caps are placed in the $x y$ lattice coordinate plane. The 'top' cap has $\Lambda_{z}=n$ and the bottom cap $\Lambda_{z}=-n$. For each cap, a complete square is generated by setting

\footnotetext{
${ }^{1}$ Finding which cell a position corresponds to is an existing function in the mesh description in OpenFOAM.
} 


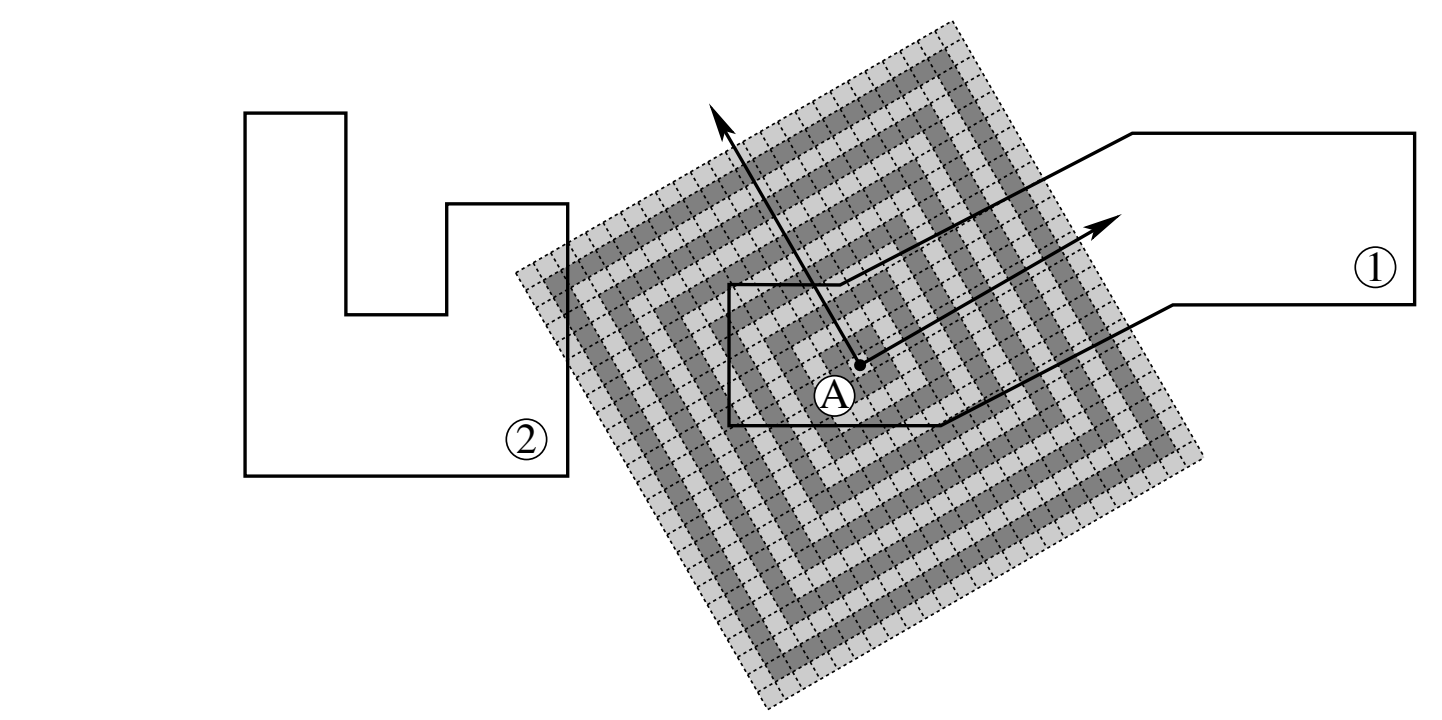

Figure 3. A single mesh zone comprising two disconnected volumes. These volumes are filled by creating an expanding cube of lattice unit cells from the anchor point, A. Volume 2 will be filled as long as volume 1 is not fully filled before the expanding cube reaches the

edge of volume 2.

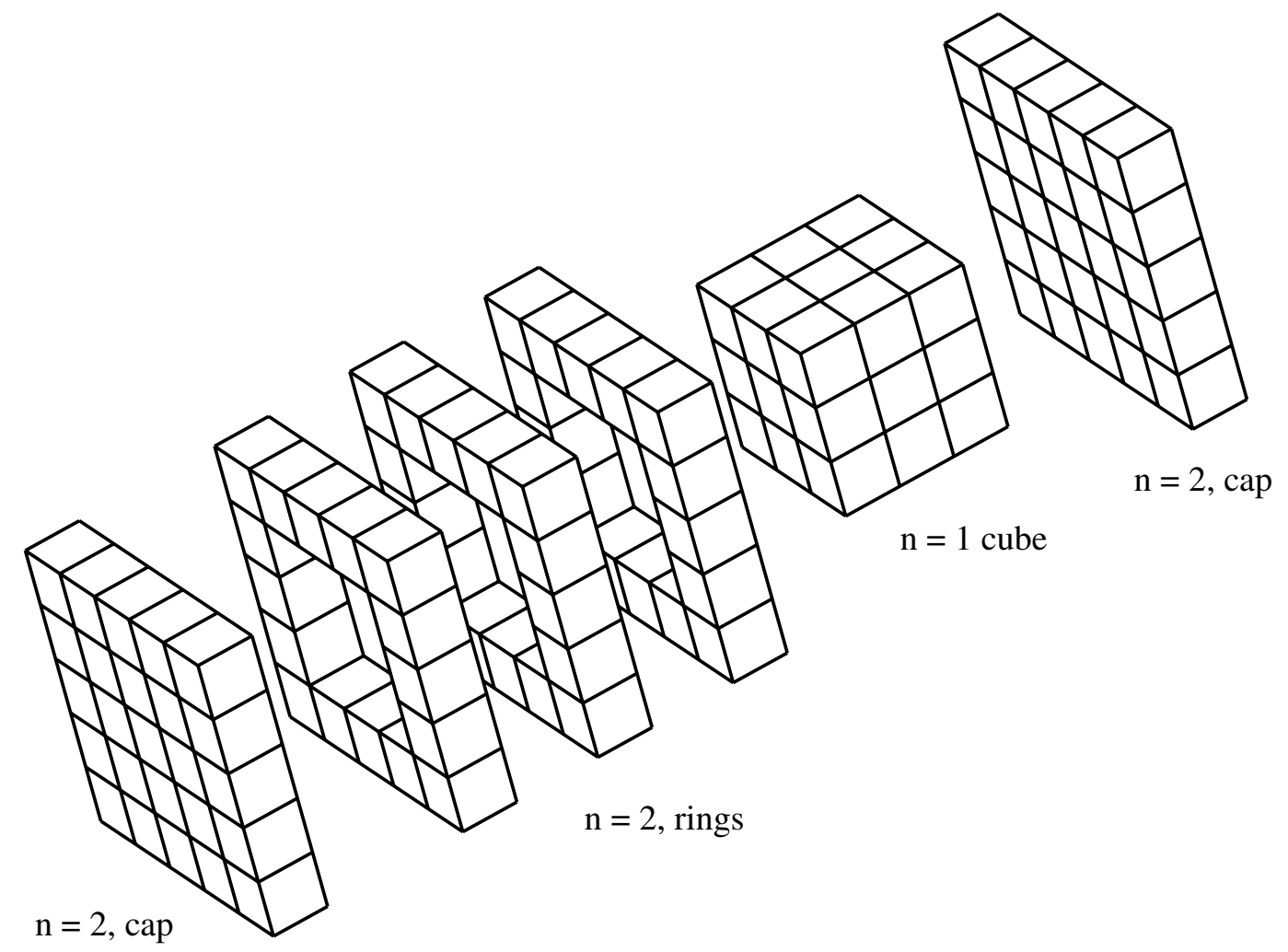

Figure 4. Exploded view of the unit cells in layer $n=2$ added to the cube of cells containing layers $n=0$ and 1. A $(2 n+1) \times(2 n+1)$ square of unit cells - a 'cap' - is placed on each end of the cells from the previous layer and $(2 n-1)$ rings are added between the caps to establish the next full layer of the cube. 


$$
\Lambda_{y}=\{-n,-n+1, \ldots, n\}
$$

and for each value of $\Lambda_{y}$ generate a line of cubes by setting

$$
\Lambda_{x}=\{-n,-n+1, \ldots, n\} .
$$

The centre unit cell $(n=0)$ is a special case and is placed at the lattice coordinate origin.

Rings. There are $2 n-1$ rings, with

$$
\Lambda_{z}=\{-n+1,-n+2, \ldots, n-1\} .
$$

The rings are created by a $2 \mathrm{D}$ expanding layer of squares algorithm. The $\left(\Lambda_{x}, \Lambda_{y}\right)$ coordinates of the unit cells to be added to the layer of the ring are generated by adding a layer to a square of unit cells. Unit cells are generated along one side of the square and replicated around the other three sides to create the whole layer. Note that this replication cannot be performed by simply rotating or reflecting the positions of molecules on one side to create the other three, because the unit cells are generally not rotationally symmetric.

The generation of an expanding square of unit cells is shown in figure 5 . The first cell $(n=0)$ is a special case and is placed at the origin of the lattice coordinate system. The coordinates of a unit cell will be given by $n$, the layer it is in, and $r$, the repetition counter: $r=\{0,1,2, \ldots, 2 n-1\}$, which generates the correct number of unit cells along each side. $\Lambda^{\mathrm{k}}$ represents the coordinates of the unit cells on the $k^{\text {th }}$ side of the layer, $k=\{1,2,3,4\}$ :

$$
\begin{aligned}
& \boldsymbol{\Lambda}^{\mathbf{1}}=(n,-n+(r+1)), \\
& \boldsymbol{\Lambda}^{\mathbf{2}}=(n-(r+1), n), \\
& \boldsymbol{\Lambda}^{\mathbf{3}}=(-n, n-(r+1)), \\
& \boldsymbol{\Lambda}^{\mathbf{4}}=(-n+(r+1),-n) .
\end{aligned}
$$

Note that the coordinate of each successive side is generated by swapping the $x$ and $y$ coordinates, negating the $y \rightarrow x$ transfer, i.e.

$$
\Lambda^{k+1}=\left(-\Lambda_{y}^{k}, \Lambda_{x}^{k}\right)
$$

\subsection{Zone specification: molConfigDict}

For each zone of cells in the mesh, the user must specify the details of the lattice to be created. This data is supplied through the molecule configuration dictionary file: molConfigDict. The entry for a zone contains the items shown in table 1.

The overall density of molecules placed in the zone (the total number of molecules divided by the total zone volume) may only be approximately correct. It will deviate slightly from the bulk value specified unless the region dimensions correspond to an integer number of unit cells and the anchor and orientation angles place and align the lattice so that the domain is filled accordingly.

It is possible to generate molecular data from an underlying, spatially-varying data field. For example, a temperature and velocity field from an initial continuum fluid mechanics simulation of the geometry 

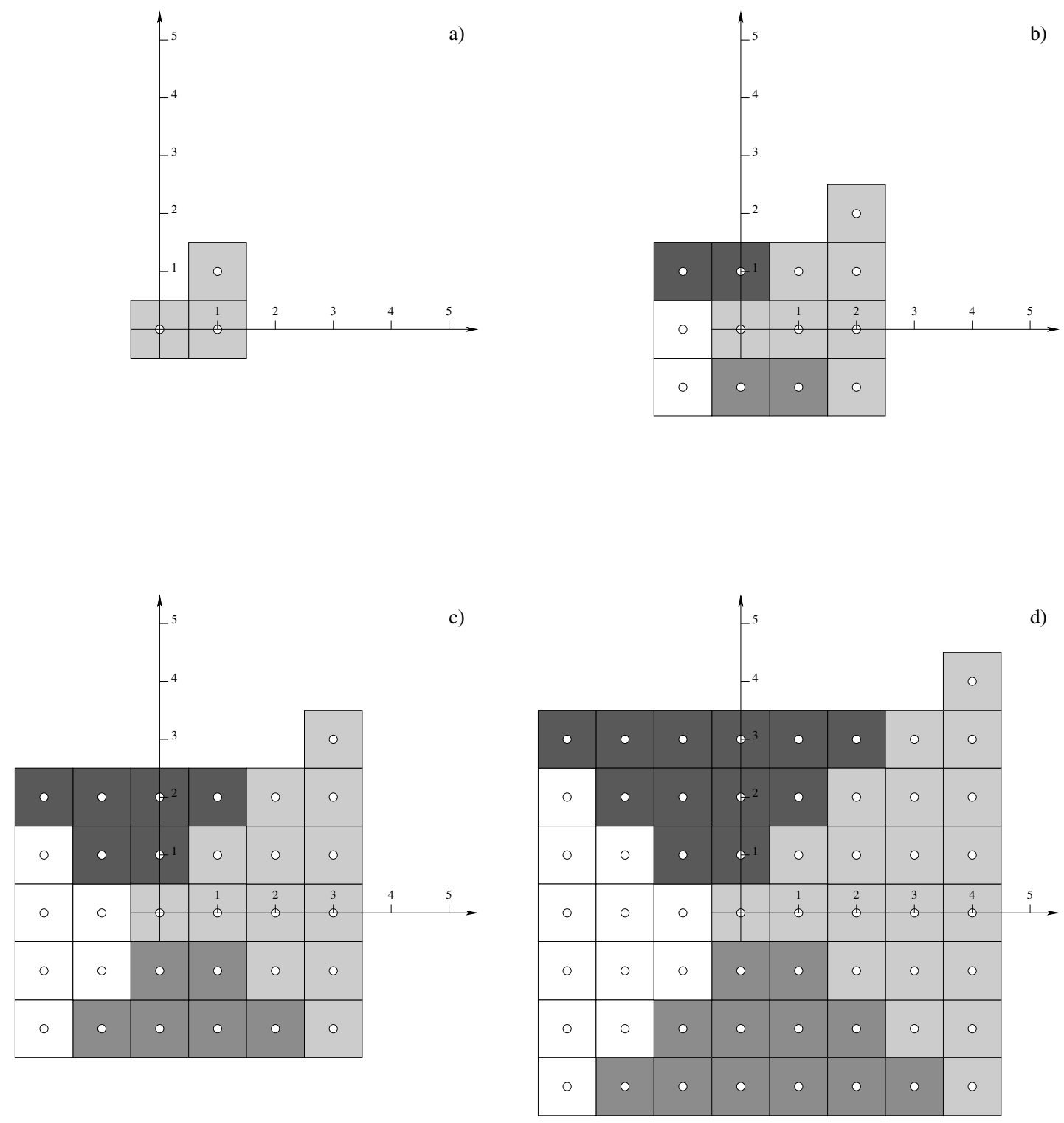

Figure 5. The construction of the sides of the square for layers $n=\{1,2,3,4\} . \boldsymbol{\Lambda}^{\mathbf{1}}$ : vertical, positive x, light-grey. $\boldsymbol{\Lambda}^{\mathbf{2}}$ : horizontal, positive $\mathrm{y}$, dark-grey. $\boldsymbol{\Lambda}^{\mathbf{3}}$ : vertical, negative $\mathrm{x}$, white. $\boldsymbol{\Lambda}^{\mathbf{4}}$ : horizontal, negative $\mathrm{y}$, mid-gray. a) $\boldsymbol{\Lambda}^{\mathbf{1}}$ for $n=1$; b) all sides for $n=1$ and $\boldsymbol{\Lambda}^{\mathbf{1}}$ for $n=2$; c) all sides for $n=2$ and $\boldsymbol{\Lambda}^{\mathbf{1}}$ for $n=3$; d) all sides for $n=3$ and $\boldsymbol{\Lambda}^{\mathbf{1}}$ for $n=4$.

Table 1. Data elements required by each zone in molConfigDict.

\begin{tabular}{lll}
\hline Entry & Description & Data Required \\
\hline $\begin{array}{l}\text { density } \\
\text { temperature }\end{array}$ & $\begin{array}{l}\text { bulk number density } \\
\text { initial temperature } \\
\text { velocityDistribution } \\
\text { choose initial velocities of molecules from, see section 2.6 }\end{array}$ & $\begin{array}{l}\text { scalar } \\
\text { scalar } \\
\text { aniform or maxwellian }\end{array}$ \\
$\begin{array}{ll}\text { average velocity to add to random velocity component } \\
\text { id }\end{array}$ & $\begin{array}{l}\text { identification of type of molecule to be added - used to de- } \\
\text { termine which intermolecular potential to use }\end{array}$ & string, e.g. LJ \\
mass of each molecule to be placed & scalar \\
latticeStructure & what structure of lattice to create & string, e.g. SC, BCC or FCC \\
anchor & the position specifying the starting point of the lattice & vector \\
anchorSpecifies & specifying whether the anchor is the position of a molecule or & molecule or corner \\
the corner of a unit cell & are the molecules to be tethered to their initial positions? & yes or no \\
the orientation of the lattice, see section 2.3 & triplet of angles \\
\hline
\end{tabular}


using OpenFOAM may be available, and the molecular velocities can be generated using the local values of temperature and bulk velocity.

To create a solid wall in a simulation it is often useful to tether molecules into a lattice, for example using a spring potential $[3,4]$, so that they retain their structure. If tethered is yes, then the initial locations of molecules in this zone will be their tether positions.

In future developments of the utility, realistic crystals will be available in a library. The id, mass and density entries will not be necessary, and additional information may be required. For example, for latticeStructure $=\mathrm{NaCl}$, molecules would be placed with the correct structure and spacing for the specified temperature (and possibly pressure), and given ids of $\mathrm{Na}$ and $\mathrm{Cl}$, with the appropriate mass and charge assigned to each.

\subsection{Generating molecule positions from a unit cell}

A rank two rotation tensor, $\mathbf{R}$, is created using the $\phi, \theta, \psi$ convention Euler angles [5] to specify the orientation of the lattice relative to the global cartesian coordinate system:

$$
\mathbf{R}=\left(\begin{array}{lll}
r_{11} & r_{12} & r_{13} \\
r_{21} & r_{22} & r_{23} \\
r_{31} & r_{32} & r_{33}
\end{array}\right)
$$

where,

$$
\begin{aligned}
& r_{11}=\cos (\psi) \cos (\phi)-\cos (\theta) \sin (\phi) \sin (\psi), \\
& r_{12}=\cos (\psi) \sin (\phi)+\cos (\theta) \cos (\phi) \sin (\psi), \\
& r_{13}=\sin (\psi) \sin (\theta), \\
& r_{21}=-\sin (\psi) \cos (\phi)-\cos (\theta) \sin (\phi) \cos (\psi), \\
& r_{22}=-\sin (\psi) \sin (\phi)+\cos (\theta) \cos (\phi) \cos (\psi), \\
& r_{23}=\cos (\psi) \sin (\theta), \\
& r_{31}=\sin (\theta) \sin (\phi), \\
& r_{32}=-\sin (\theta) \cos (\phi), \\
& r_{33}=\cos (\theta) .
\end{aligned}
$$

Another rank two tensor, $\mathbf{G}$, is required to specify the scaling of the cubic unit cells to the shape of the lattice,

$$
\mathbf{G}=\left(\begin{array}{ccc}
g_{11} & 0 & 0 \\
0 & g_{22} & 0 \\
0 & 0 & g_{33}
\end{array}\right)
$$

This is specific to the lattice structure used and the density of the crystal. $\mathbf{R}$ and $\mathbf{G}$ are used in conjunction with the lattice anchor, $\mathbf{A}$, to transform a position in lattice coordinates, $\boldsymbol{\Lambda}$, to a position, $\mathbf{P}$, in the global coordinate system

$$
\mathbf{P}=\mathbf{A}+\mathbf{R} \cdot(\mathbf{G} \cdot \mathbf{\Lambda})
$$


Each lattice type will place its own number of molecules in the appropriate positions around the unit cell centre. Three examples given below are the simple, body-centred and face-centred cubic lattices where

$$
g_{11}=g_{22}=g_{33}=g
$$

because the lattices are cubic. These examples are based on lattice generation steps in [6].

2.3.1 Simple cubic $(S C)$. For $\rho$, the user specified bulk number density

$$
g=\rho^{-1 / 3}
$$

and if anchorSpecifies $=$ molecule then a single molecule is placed at the unit cell centre, $\boldsymbol{\Lambda}$. If anchorSpecifies $=$ corner the molecule is shifted in the lattice coordinates to $\boldsymbol{\Lambda}-(0.5,0.5,0.5)$ prior to transformation by equation (8).

2.3.2 Body centred cubic $(\boldsymbol{B C C})$. For a unit cell centre $\boldsymbol{\Lambda}$, if anchorSpecifies $=$ molecule, molecules are placed at

$$
\begin{gathered}
\left(\Lambda_{x}, \Lambda_{y}, \Lambda_{z}\right), \\
\left(\Lambda_{x}+0.5, \Lambda_{y}+0.5, \Lambda_{z}+0.5\right),
\end{gathered}
$$

in lattice coordinates, then transformed to the global coordinate system using equation (8), where

$$
g=\left(\frac{\rho}{2}\right)^{-1 / 3}
$$

If anchorSpecifies $=$ corner then the two positions above are shifted in the lattice coordinates by $(-0.25,-0.25,-0.25)$ prior to transformation.

2.3.3 Face centred cubic $(\boldsymbol{F C C})$. For a unit cell centre $\boldsymbol{\Lambda}$, if anchorSpecifies $=$ molecule, molecules are placed at

$$
\begin{gathered}
\left(\Lambda_{x}, \Lambda_{y}, \Lambda_{z}\right), \\
\left(\Lambda_{x}, \Lambda_{y}+0.5, \Lambda_{z}+0.5\right), \\
\left(\Lambda_{x}+0.5, \Lambda_{y}, \Lambda_{z}+0.5\right), \\
\left(\Lambda_{x}+0.5, \Lambda_{y}+0.5, \Lambda_{z}\right),
\end{gathered}
$$

in lattice coordinates, then transformed to the global coordinate system using equation (8), where

$$
g=\left(\frac{\rho}{4}\right)^{-1 / 3}
$$

If anchorSpecifies $=$ corner then the four positions above are shifted in the lattice coordinates by $(-0.25,-0.25,-0.25)$ prior to transformation. 


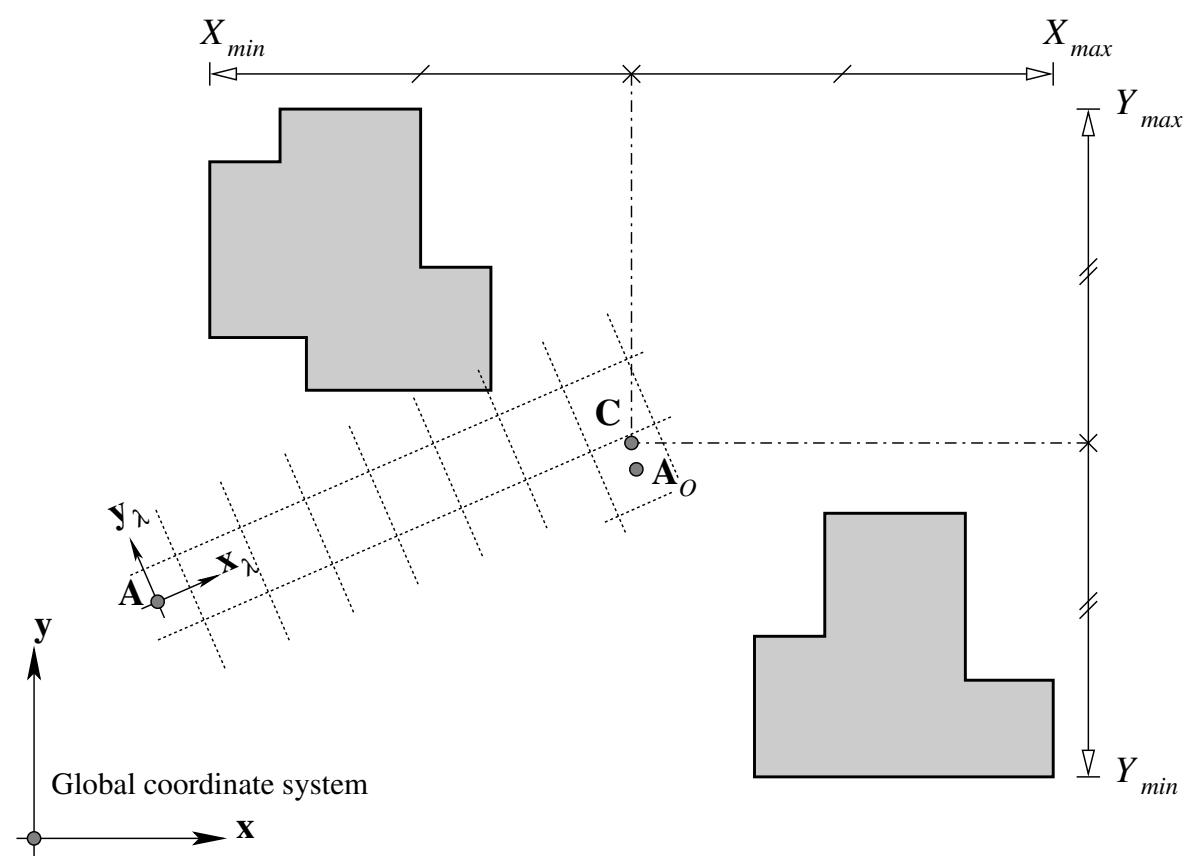

Figure 6. Placing the optimised anchor at the unit cell position closest to the centre of the volumes in the zone.

\subsection{Optimising the anchor position}

The choice of anchor strongly influences the speed of the algorithm An anchor near to the centre of the zone volume minimises the number of unnecessary unit cells to be tested. This has a large impact when the mesh has been decomposed for parallelisation because the anchor may lie far from the spatial region assigned to the processor in question. The algorithm will test many unnecessary unit cells before it reaches the region that the processor in question has been assigned, whereupon the expanding cube will be large and only a small proportion of the unit cells added to a layer will create molecules that are accepted.

The anchor position can also impact on the ability to fill disconnected volumes. If a volume of a zone finishes filling before another starts, then the algorithm will terminate and not fill the further away volume. Again, an anchor near the centre of the zone mitigates this. Both of these problems are addressed by automatically moving the anchor from the user-specified point to the lattice site that is closest to the zone volume centre; molecules are created at the same positions as they are when starting from the user-specified anchor, so no change in the configuration results.

The centroid of the volume is not the quantity of interest because this is weighted towards larger volumes. The required centre is instead calculated by finding the midpoint between the extremities of the volumes of the zone in the global coordinate system,

$$
\mathbf{C}=\frac{1}{2}\left(X_{\min }+X_{\max }, Y_{\min }+Y_{\max }, Z_{\min }+Z_{\max }\right) .
$$

The closest lattice point to $\mathbf{C}$ is required, and will be called the optimised anchor, $\mathbf{A}_{O}$ (see figure 6). Rewriting equation (8) for $\mathbf{C}$, where $\boldsymbol{\Lambda}_{C}$ is the position of the volume centre in local lattice coordinates and $\mathbf{A}$ is the user specified anchor,

$$
\mathbf{C}=\mathbf{A}+\mathbf{R} \cdot\left(\mathbf{G} \cdot \boldsymbol{\Lambda}_{C}\right),
$$

and rearranging for $\boldsymbol{\Lambda}_{C}$, 
Table 2. Pertinent molConfigDict entries for the three zone test case. Note that the orientation angles are specified in degrees.

\begin{tabular}{|c|c|c|c|}
\hline Entry & Bottom Wall & Liquid & Top Wall \\
\hline $\begin{array}{l}\text { density } \\
\text { bulkVelocity } \\
\text { id } \\
\text { latticeStructure } \\
\text { anchor } \\
\text { anchorSpecifies } \\
\text { tethered } \\
\text { orientationAngles }\end{array}$ & $\begin{array}{l}0.8 \\
\left(\begin{array}{lll}0.0 & 0.0 & 0.0\end{array}\right) \\
\text { WALLB } \\
\text { FCC } \\
\left(\begin{array}{lll}16.25 & 4.75 & 6.25\end{array}\right) \\
\text { molecule } \\
\text { yes } \\
\left(\begin{array}{lll}30 & 0 & 0\end{array}\right)\end{array}$ & $\begin{array}{l}0.8 \\
\left(\begin{array}{lll}2.0 & 0.0 & 0.0\end{array}\right) \\
\text { LJ } \\
\text { SC } \\
\left(\begin{array}{lll}6.25 & 13.25 & 6.25\end{array}\right) \\
\text { molecule } \\
\text { no } \\
\left(\begin{array}{lll}45 & 0 & 0\end{array}\right)\end{array}$ & 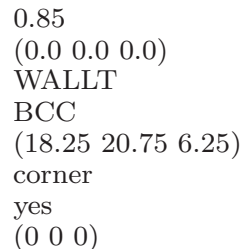 \\
\hline
\end{tabular}

$$
\boldsymbol{\Lambda}_{C}=\mathbf{G}^{-\mathbf{1}} \cdot\left(\mathbf{R}^{-\mathbf{1}} \cdot(\mathbf{C}-\mathbf{A})\right) .
$$

$\mathbf{R}$ is orthogonal, so $\mathbf{R}^{-\mathbf{1}}=\mathbf{R}^{\mathbf{T}}$ and

$$
\mathbf{G}^{-\mathbf{1}}=\left(\begin{array}{ccc}
1 / g_{11} & 0 & 0 \\
0 & 1 / g_{22} & 0 \\
0 & 0 & 1 / g_{33}
\end{array}\right)
$$

The closest unit cell centre in lattice coordinates, $\boldsymbol{\Lambda}_{A_{O}}$, is found by

$$
\boldsymbol{\Lambda}_{A_{O}}=\left(\operatorname{nint}\left(\Lambda_{C x}\right), \operatorname{nint}\left(\Lambda_{C y}\right), \operatorname{nint}\left(\Lambda_{C z}\right)\right),
$$

where nint is a function returning the nearest integer to the argument. The optimised anchor is given finally by

$$
\mathbf{A}_{O}=\mathbf{A}+\mathbf{R} \cdot\left(\mathbf{G} \cdot \boldsymbol{\Lambda}_{A_{O}}\right),
$$

and $\mathbf{A}_{O}$ is used instead of $\mathbf{A}$ in equation (8) when generating unit cells.

Optimising the anchor position does not guarantee that all possible arrangements of disconnected volumes will be filled, although it substantially increases the number that will be. If a volume in a particular zone does not get filled with molecules, then the zone can be subdivided and the new zones given identical details in molConfigDict. The mesh can be redecomposed to distribute the cells amongst processors differently if the problem occurs only in parallel.

\section{$2.5 \quad$ A three zone test case}

The operation of the placement algorithm is illustrated by the example case illustrated in figure 7 . Table 2 shows the pertinent molConfigDict entries describing the configuration of the system. Three different types of molecule are created, in three different lattice structures. The wall zones tether their molecules in place and the liquid region is given an initial average velocity. All numerical values are expressed in MD reduced units $[6,7]$ with an energy scale of $120 k_{b}$, where $k_{b}$ is the Boltzmann constant, a length scale of $0.34 n m$ and a mass scale of $6.6904 \times 10^{-26} \mathrm{~kg}$. In future versions of molConfig and the MD solver [2] the user will specify input parameters in SI units and the code will convert these to reduced units for internal use only.

Figure 8 shows the configuration of molecules that molConfig generates and the positions of the userspecified and optimised anchors. The bottom wall anchor is in the optimal position. The bottom wall and liquid anchors are positioned on top of molecules and the top wall anchors are between molecules, as per their respective anchorSpecifies entries. Figure 9 shows the molecules in 3D. 


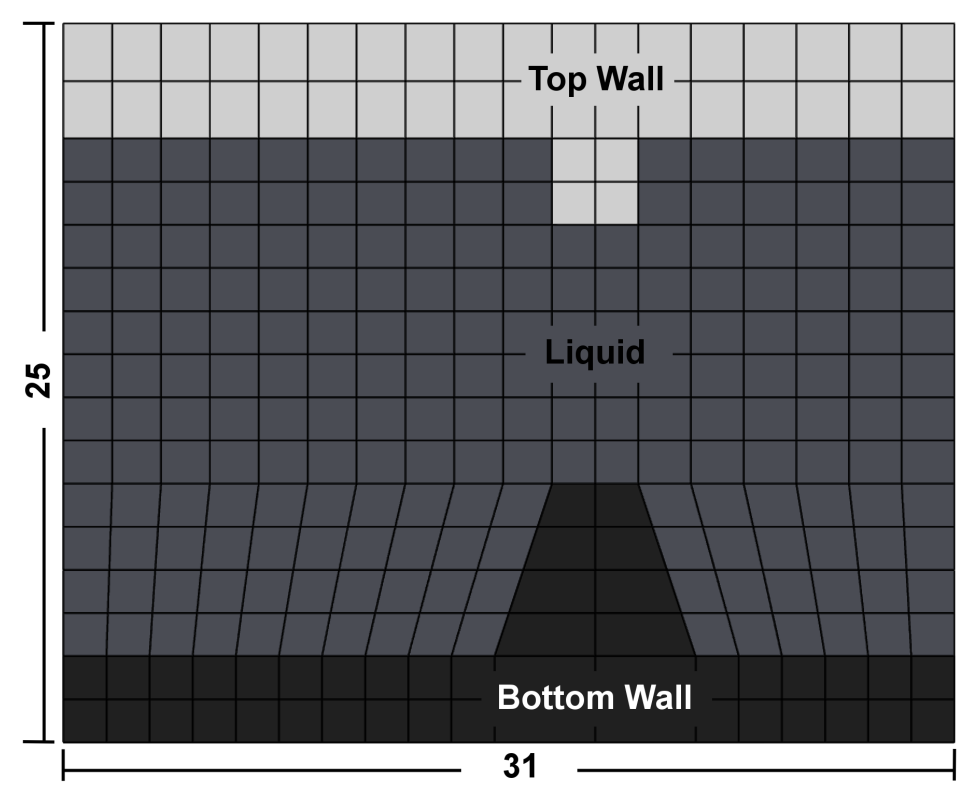

Figure 7. Three zone test case mesh and zones: a channel with a constriction. Overall dimensions are $31 \times 25 \times 12 \mathrm{MD}$ reduced units.

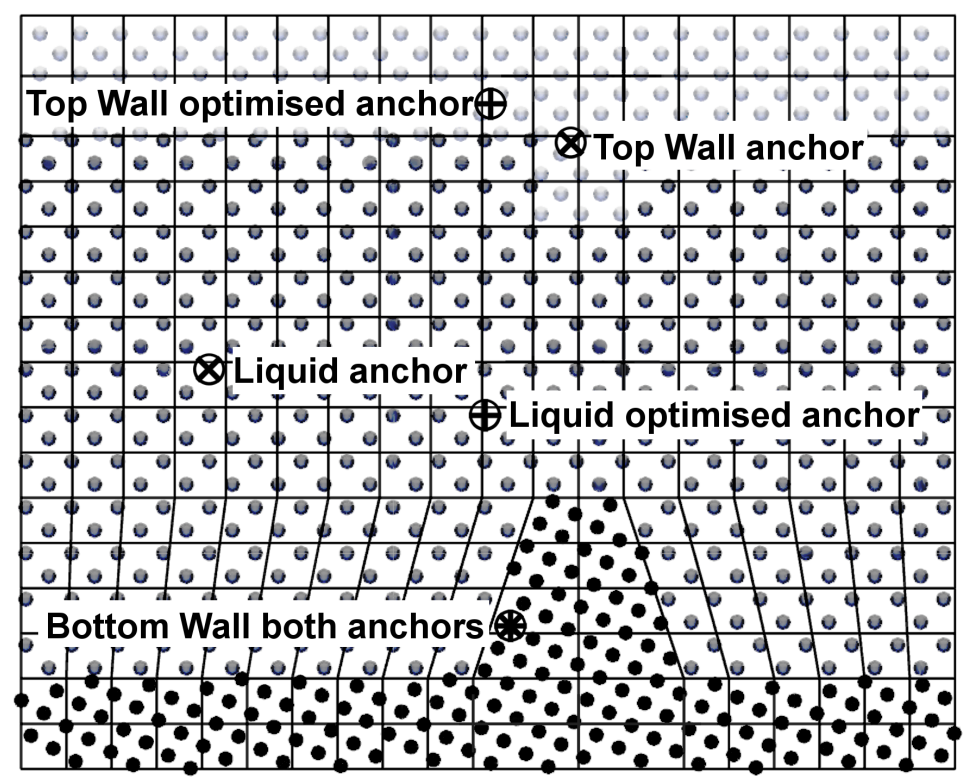

Figure 8. Three zone test case configuration of molecules. Molecules coloured according to id. User specified anchors $\otimes$ and optimised anchors $\oplus$ are shown.

The mesh is decomposed into four portions for parallelisation using the METIS [8] library (see figure 10). The mesh portions assigned to processors 1 and 3 contain disconnected volumes belonging to the same zone. Focusing on the portion assigned to processor 1 (see figure 11) there are two disconnected volumes each of the bottom wall and liquid zones. The optimised anchors for these two zones are shown. The same configuration of molecules is generated when the four portions are filled independently by different processors, as when the whole mesh is filled using one processor. Figures 7 to 11 were generated using ParaView [9].

\subsection{Molecular velocity generation}

The velocities of the molecules created are initialised to a set of thermal (random) velocities to match the user defined temperature, $T_{U}$, in the molConfigDict entry for the zone. The equilibrium kinetic tempera- 


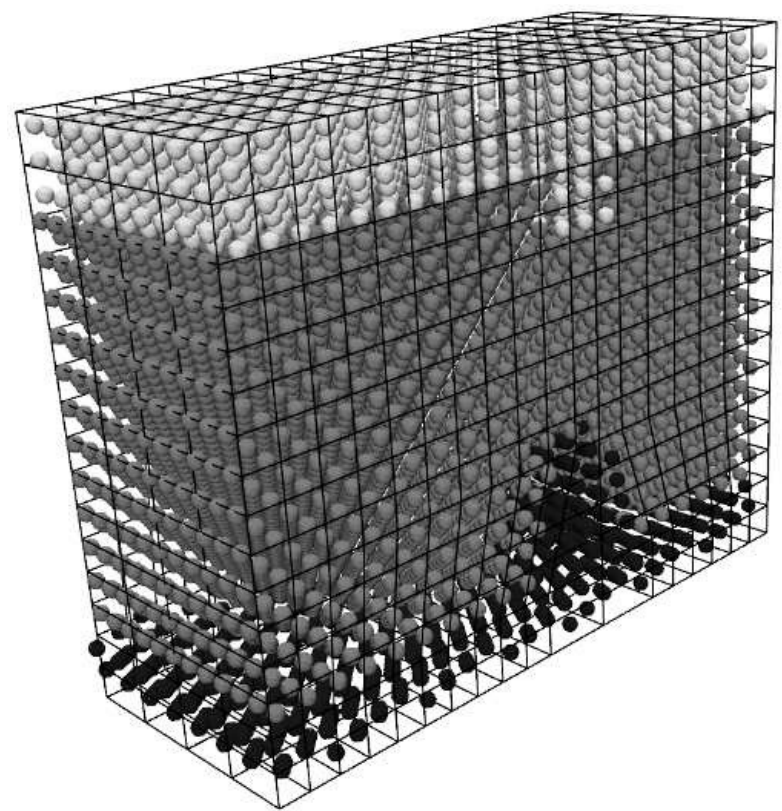

Figure 9. 3D view of the molecules created in the three zone test case. Molecules coloured according to id.

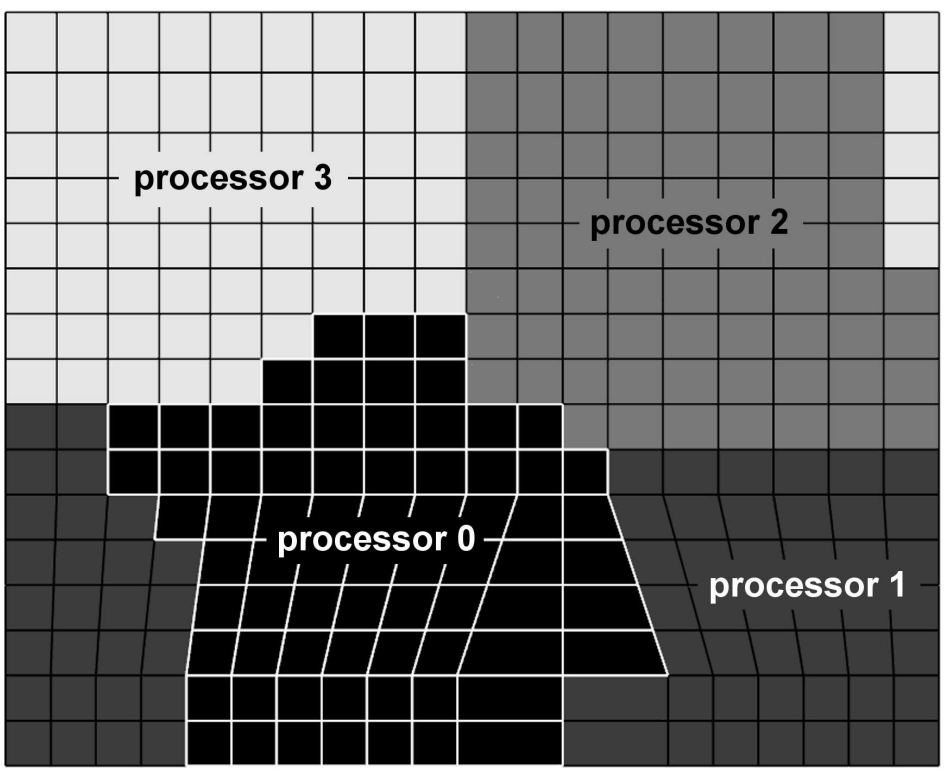

Figure 10. The mesh for the three zone test case decomposed into 4 portions for parallelisation. Processors 1 and 3 have zones with disconnected volumes.

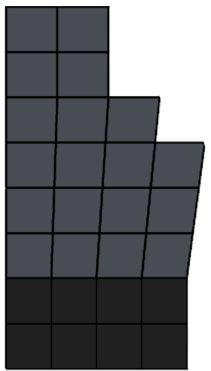

$\bigoplus$

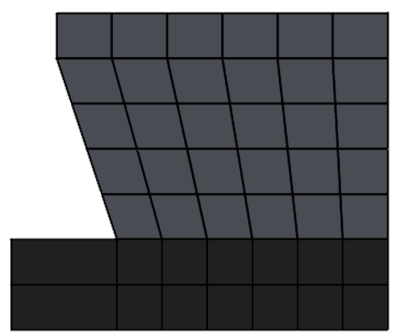

Figure 11. The disconnected volumes of the bottom wall and liquid zones residing on processor 1 . The shading corresponds to the zones as shown in figure 7 . The optimised anchors for filling each zone on this processor are shown. 
ture, $T$, of a stationary system of $N_{M}$ molecules [7] is then given by

$$
T=\frac{1}{3 N_{M}} \sum_{i=1}^{N_{M}} m_{i} v_{i}^{2},
$$

in MD reduced units, where $m_{i}$ is the mass of a molecule and $v_{i}$ is the magnitude of its velocity. The direction of the velocity assigned to each molecule is random, and the magnitude of each is chosen to give the correct temperature. The distribution of molecular speeds can either be uniform or Maxwellian.

2.6.1 Uniform distribution. A vector, $\mathbf{v}_{r}$, is created for each molecule which has each of its components generated by a uniform random number generator returning a value between -1 and 1 . The velocity assigned to the molecule, $\mathbf{v}_{i}$, is given by

$$
\mathbf{v}_{i}=\sqrt{\frac{3 T_{U}}{m_{i}}} \frac{\mathbf{v}_{r}}{\left|\mathbf{v}_{r}\right|}
$$

The prefactor is obtained by rearranging equation (15) for $v_{i}$ with $N_{M}=1$. A uniform initial velocity distribution can be useful to determine when a system has equilibrated: measuring the velocity distribution in the system as the simulation progresses and identifying when it reaches a Maxwellian form.

2.6.2 Maxwellian distribution. The equilibrium (Maxwellian) velocity distribution of a system is defined as the probability of a component of a molecule's velocity in any direction being a normal distribution with a zero mean [10], i.e.

$$
P\left(v_{x}\right)=\frac{1}{\sigma \sqrt{2 \pi}} e^{-v_{x}^{2} / 2 \sigma^{2}}
$$

where $\sigma=\sqrt{T / m}$. The velocity to assign to a molecule, $\mathbf{v}_{i}$, is obtained by sampling the component in each direction from a normal distribution random number generator with zero mean and variance $\sigma^{2}=T_{U} / m_{i}$.

2.6.3 Remove drift velocity and apply average. The finite number of random numbers used to generate the molecular velocities will in general not result in a mean velocity of zero. This remaining 'drift' velocity is removed and the user-specified average velocity for the zone in question, $\mathbf{v}_{U}$, is added to each molecule by

$$
\mathbf{v}_{i}^{\prime}=\mathbf{v}_{i}-\frac{1}{N} \sum_{i=1}^{N} \mathbf{v}_{i}+\mathbf{v}_{U}
$$

\subsection{Avoiding high energy overlaps at zone boundaries}

At the interface between different zones it is possible that molecules will be placed so close to each other that the high-energy, repulsive portions of their intermolecular potentials will overlap. This causes the MD simulation to crash because these high energy overlaps accelerate molecules to very large velocities in the first timestep, where they then overlap with the first molecule that they collide with (due to the finite timestep), accelerating it to a very high velocity. This causes a cascade of high energy impacts and the total energy in the simulation to increase uncontrollably. 


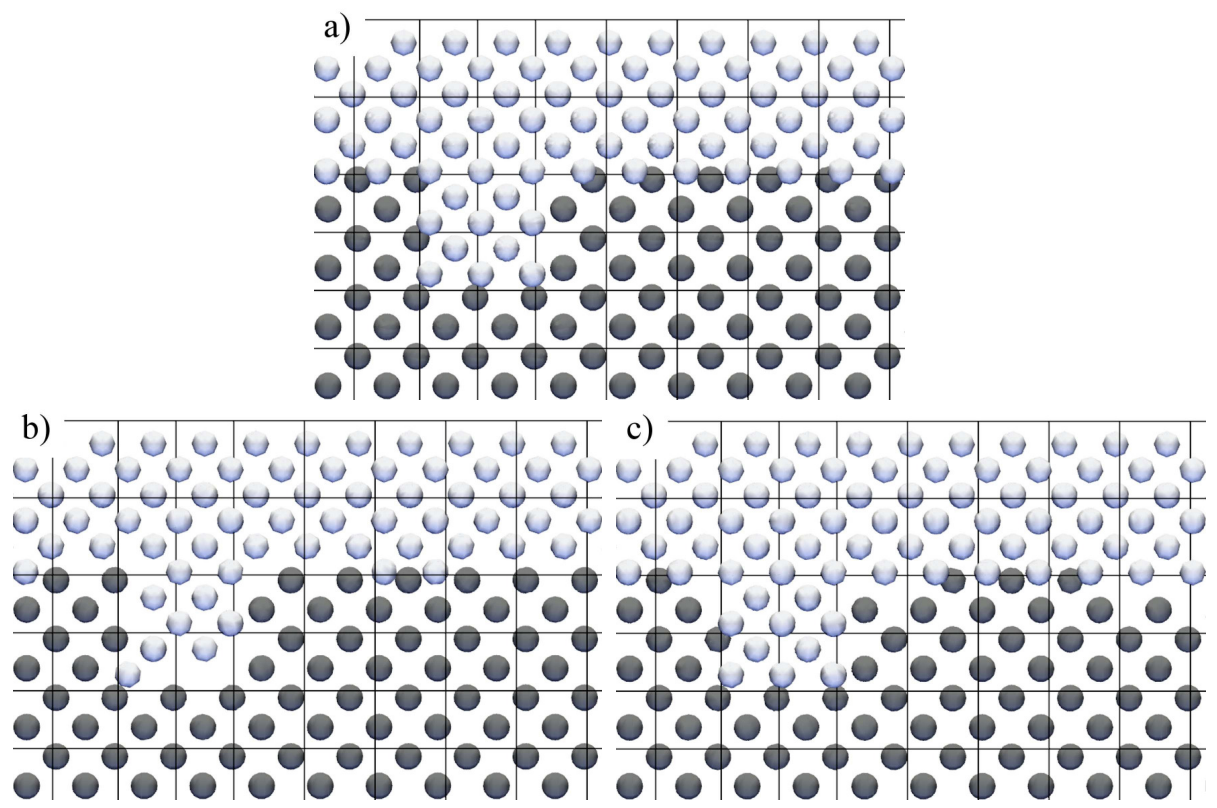

Figure 12. A close-up of the top right of figure 8 showing the constriction in the top wall zone. a) shows the molecules created by filling the zones, note the overlapping molecules at the interface. b) the result when WALLT comes before LJ (see table 2) in the removal order list: liquid zone molecules (LJ) are retained at the expense of top wall molecules (WALLT). c) the result when LJ comes before WALLT in the removal order list: top wall molecules (WALLT) are retained at the expense of liquid zone molecules (LJ).

This problem is avoided by calculating the intermolecular energy between pairs of molecules [2] before the first timestep and any pairs sharing a potential energy above a user defined threshold are identified. If the molecules in a high energy pair have the same id (are the same type of molecule) then one of them is deleted from the simulation. If the molecules have different ids then a user specified removal order list is consulted. The molecule that appears first in the removal order list is deleted. An example of this is shown in figure 12. This also applies across periodic and interprocessor boundaries, where molecules on either side of these boundaries can be so close to each other that they overlap.

2.7.1 Insertion of complex molecules. The high energy overlap removal mechanism can be used as the basis for the insertion of complex molecules. For example, the insertion of large biomolecules into a system, or adding carbon nanotubes spanning a solid barrier between two fluid reservoirs. The complex molecule can be created in place and, providing that the solid and solvent molecules appear before any of the constituents of the complex molecule on the removal order list, it will be embedded into the existing matter.

\section{Computational performance}

A cubic test system of 50 units side length (in MD reduced units) was chosen to evaluate the performance of molConfig. The commercial CFD meshing software GAMBITßwas used to generate 21 different tetrahedral meshes with these dimensions. They were generated by specifying the number of cell edges to be placed on the edges of the bounding geometry, and then allowing GAMBITß to automatically generate a tetrahedral mesh. Edge numbers of 10-30 were used and the number of cells, $N_{C}$, generated in each case shown in table 3. An example mesh (edge number 15) is shown in figure 13. A single zone was defined for the whole mesh and filled with an SC lattice with five different densities, $\rho=\{0.8,0.4,0.2,0.08,0.02\}$, creating $N_{M}=\{97336,46656,27000,10648,2744\}$ molecules in each mesh. The time taken for molConfig to fill each mesh was recorded and is shown in figure 14. Linear trends fit the computational time data very well, although the negative intercepts of the fitted lines indicate that linear scaling does not hold when there are very few cells. All timing tests were performed on a $\mathrm{PC}$ with a $2.8 \mathrm{GHz}$, AMD Athlon ${ }^{\mathrm{TM}} \mathrm{FX}-62$ processor.

Each line in figure 14 represents a constant number of molecules with a varying number of cells in the 
Table 3. Number of cells in test tetrahedral meshes generated by GAMBIT®.

\begin{tabular}{ccccccccccc}
\hline $\begin{array}{c}\text { edge no. } \\
N_{C}\end{array}$ & 10 & 11 & 12 & 13 & 14 & 15 & 16 & 17 & 18 & 19 \\
& 7520 & 9748 & 11101 & 19344 & 22289 & 22338 & 28113 & 36646 & 43420 & 50877 \\
\hline 20 & 21 & 22 & 23 & 24 & 25 & 26 & 27 & 28 & 29 & 30 \\
56171 & 67520 & 73801 & 88334 & 97488 & 105385 & 129761 & 145016 & 145196 & 185016 & 196847 \\
\hline
\end{tabular}

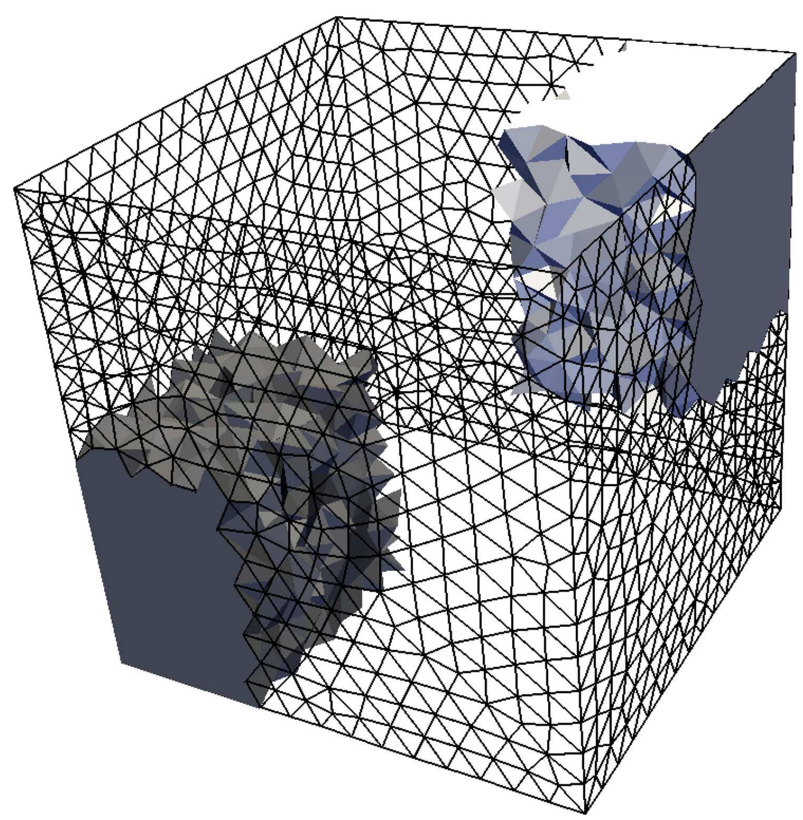

Figure 13. Tetrahedral test mesh with edge number 15. The outline of cells on four of the six faces of the bounding cube are shown, and two of the twelve portions of cells are shown from the mesh when decomposed for parallelisation.

mesh. The dependence of the computational time on the number of molecules is examined by holding the number of cells constant, as shown in figure 15 for three meshes. The three vertical lines on figure 14 correspond to the meshes chosen. This data does not fit a linear trend; the computational cost grows more slowly than a linear relationship with increasing number of molecules in a fixed mesh. A function of the form:

$$
p+q\left(N_{M} / r\right)^{s},
$$

where $p, q, r$ and $s$ are the fitting parameters, was found to fit the data well, with $r$ and $s$ similar for each line.

In a practical system, the computational cost will increase as approximately the 'problem size' squared, because a larger problem will involve a larger mesh with more cells, scaling $O\left(N_{C}\right)$, as well as a larger number of molecules, scaling approximately $O\left(N_{M}\right)$.

\subsection{Aspect ratio}

The speed of molecule generation is highly dependent on the shape of the mesh; for the cubic lattices used here, cubic domains are the fastest to fill because they match the shape of the expanding volume of unit cells. Any deviation from cubic takes longer to fill for the same number of cells and molecules. To examine this, a cuboid regular mesh of $20 \times 20 \times 20$ cells was created with various aspect ratios, see figure 16 . The number of cells and the volume of the system was held constant to give approximately ${ }^{1}$ the same number of molecules, $N_{M}$. For a cuboid with a base area of $B^{2}$, and a side length of $L$, the volume is

\footnotetext{
${ }^{1}$ The number of molecules varies because the geometry for a particular aspect ratio does not exactly match the geometry of the lattice that it is being filled with. However, the maximum deviation from, in this case, 64000 molecules is only 496 , or $0.775 \%$.
} 


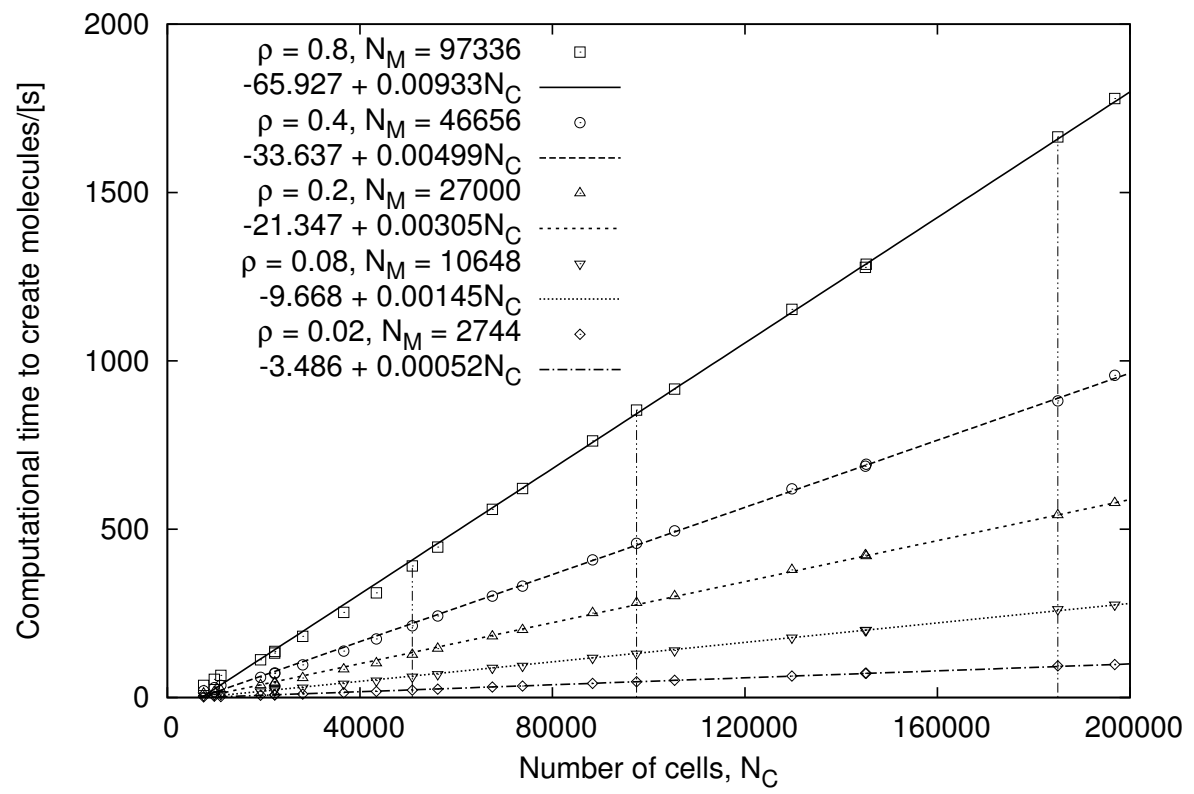

Figure 14. The computational time required to generate a given number of molecules with a varying number of cells in the mesh. The vertical lines are the meshes selected for figure 15.

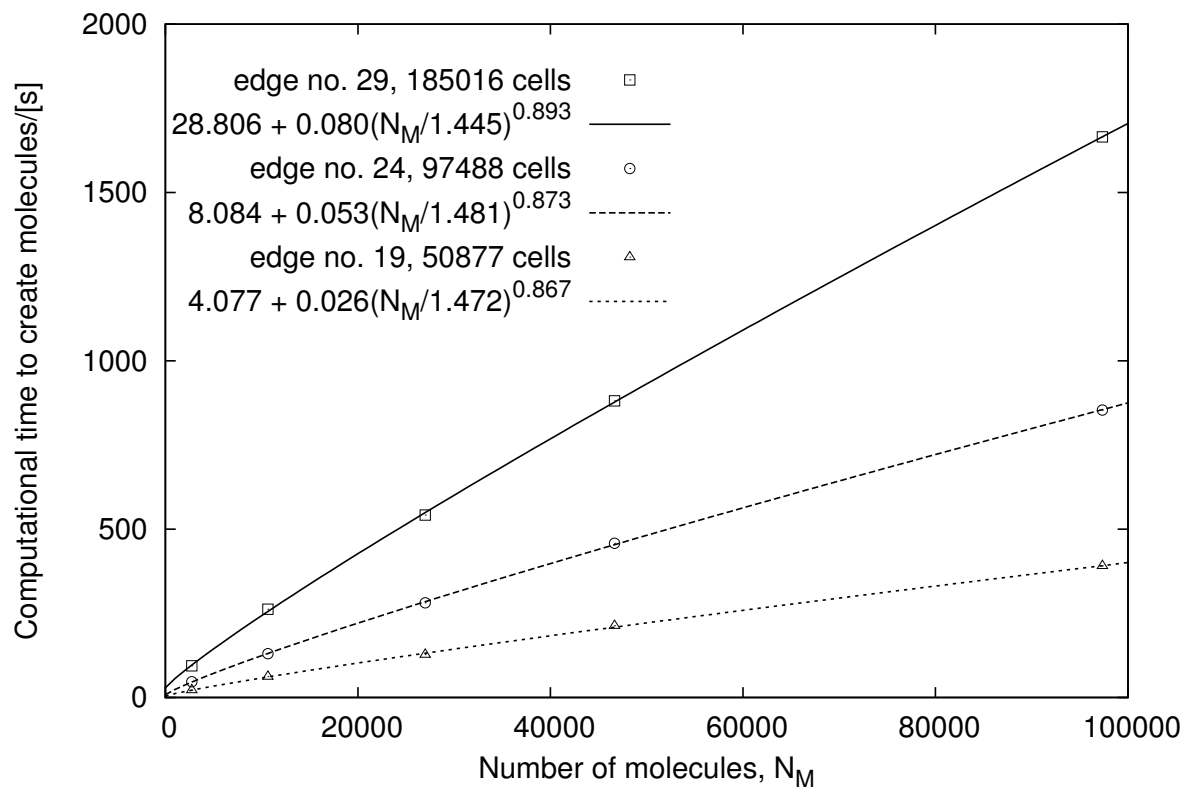

Figure 15. The computational time required to fill a mesh of a given number of cells with a varying number of molecules.

$$
V=B^{2} L
$$

Defining the aspect ratio, $a$, of the system to be

$$
a=\frac{L}{B}
$$

substituting this into equation (19), and rearranging for $a$ gives 


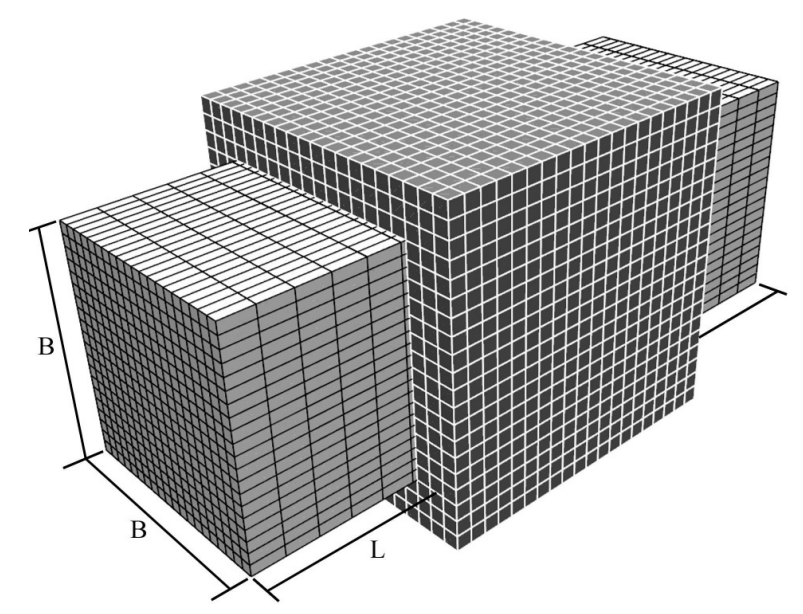

Figure 16. Two examples of the mesh used for examining aspect ratio sensitivity: $a=1$ (dark, white grid) and $a=(40 / 28)^{3}=2.915$ (light, black grid). Note that the number of cells is the same in both cases (20 in each direction); they are stretched to match the overall dimensions.

Table 4. The performance of molConfig for a cuboid system with varying aspect ratio.

\begin{tabular}{rccccccccccc}
\hline$B$ & 40 & 38 & 36 & 34 & 32 & 30 & 28 & 26 & 24 & 22 & 20 \\
\hline$a$ & 1 & 1.166 & 1.371 & 1.628 & 1.953 & 2.370 & 2.915 & 3.641 & 4.630 & 6.011 & 8 \\
$N_{M}$ & 64000 & 63888 & 63936 & 64220 & 64288 & 63900 & 64304 & 63580 & 63504 & 63534 & 64000 \\
time/[s] & 17 & 38 & 74 & 120 & 176 & 300 & 450 & 687 & 1186 & 1959 & 3433 \\
\hline
\end{tabular}

$$
a=\left(\frac{B_{1}}{B}\right)^{3}
$$

where $B_{1}$ is the side dimension when $a=1$, used to set the total system volume. Here $B_{1}=40$, so $V=64000$. Table 4 lists the aspect ratios used, the number of molecules generated and the computational time required to fill each of the meshes with an $\mathrm{SC}$ lattice of density $=1$. The computational time taken for each aspect ratio, relative to the time required for $a=1$, is shown in figure 17. A quadratic function fits the data very well. High aspect ratio mesh shapes incur a significant computational time penalty with the expanding cube volume filling-algorithm because unnecessary unit cells will be tested in a large volume outside the mesh.

\section{$3.2 \quad$ Parallel performance.}

The parallel performance of molConfig has not been subjected to timing tests because the processors do not need to communicate during the filling process. Any deviation from ideal speedup for parallel molecule generation will be mostly determined by the size and shape of the portions of the mesh that result from spatial decomposition. For example, a cubic system divided into 8 smaller, equally sized cubes and filled on 8 processors is likely to fill faster than the same cubic system divided into 12 strips and filled on 12 processors.

\section{Conclusions}

The ability to generate configurations of molecules directly within mesh geometries created by widely used engineering CAD tools means that complex, realistic systems can be set-up and simulated quickly. This is also a significant advantage for hybrid continuum-MD simulations (e.g. see [11]) in realistic geometries, where a domain of any shape can constitute the MD portion of the simulation. 


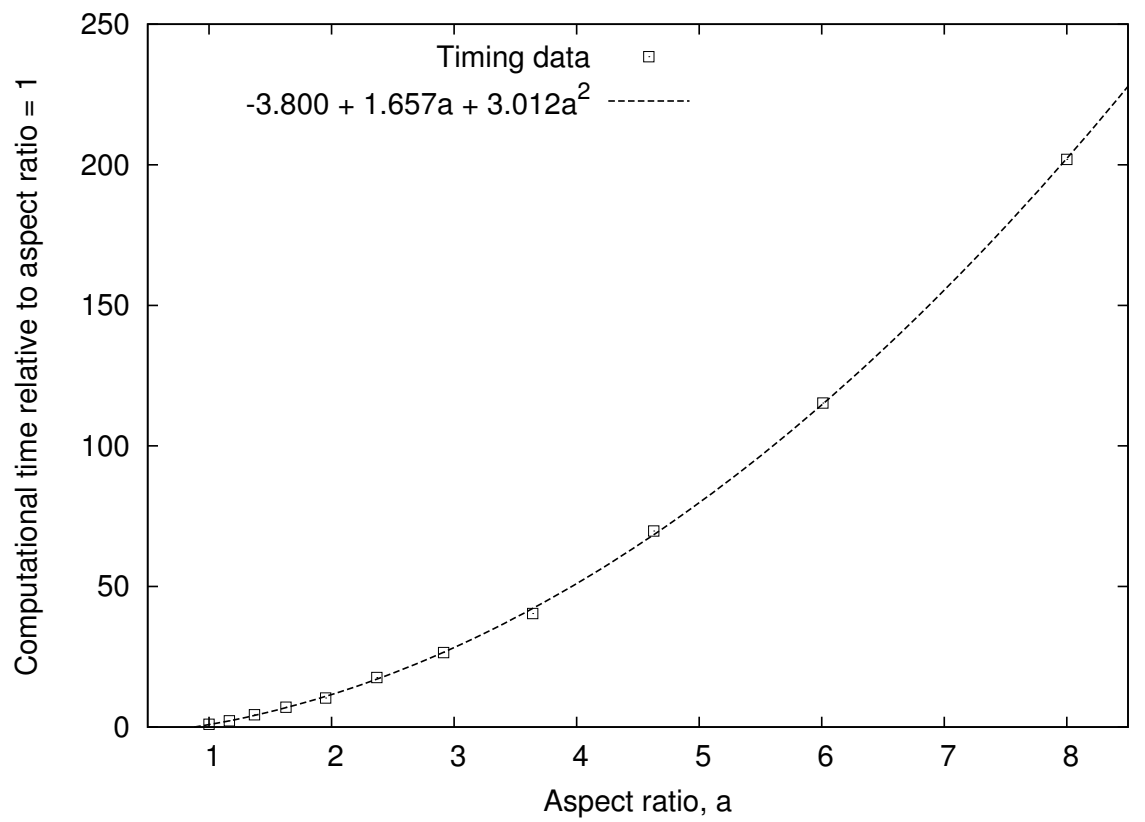

Figure 17. The computational cost of filling varying aspect ratio cuboids, relative to the cost of a cubic system of the same volume. A quadratic dependence is observed.

\subsection{Choosing mesh dimensions and anchor positions}

The overall dimensions and refinement of the mesh will be usually dictated by concerns other than the speed of molecule generation: the impact on the speed of intermolecular force calculation and the resolution of flow properties, for example [2]. The scaling of the molConfig algorithm is linear with the number of cells in the mesh and better than linear with the number of molecules, so the molecule generation process does not impose unreasonable constraints on the choice of mesh. Very high aspect ratio shapes (long channels, for example) present a performance penalty, and should be subdivided into several smaller zones to speed up molecule generation. Placing the anchor for all of these zones at the same place will ensure that they are filled with a single crystal; the anchor will be optimised for each zone automatically.

Choosing the dimensions of the domain requires care if a solid crystal wall extends across a periodic boundary; the domain must be sized to permit an integer number of lattice unit cells in the wall zone to prevent gaps or overlaps in the lattice. The anchor of the lattice should be chosen so that a plane of a lattice does not lie exactly along the surface of the mesh. If this occurs, then numerical round off errors will cause patchy placement of molecules in this plane - some molecules will be inside the domain and some outside. A small shift of the anchor will move the plane off the surface and prevent this. A similar issue occurs when generating molecules in parallel: if a molecule lies exactly on a face shared by two processors, each processor may place a molecule in essentially the same place. This is more difficult to prevent, because it depends on how the mesh is decomposed, but does not cause problems because one of the duplicated molecules will be removed by the high energy overlap check.

\subsection{Future developments}

To expand the range of materials that can be generated by molConfig, all fourteen Bravais lattices [12] should be incorporated to allow real, non-homogeneous crystals to be filled into volumes. A library of common solids and liquids draft from the open literature should be established.

\section{$5 \quad$ Acknowledgements}

The authors would like to thank Chris Greenshields of Strathclyde University, and Henry Weller and Mattijs Janssens of OpenCFD Ltd. for useful discussions. This work is funded in the UK by Strathclyde 
University, the Miller Foundation and the James Weir Foundation, and through a Philip Leverhulme Prize for JMR from the Leverhulme Trust.

\section{References}

[1] OpenFOAM: The Open Source CFD Toolbox. http://www.openfoam.org.

[2] Graham B. Macpherson and Jason M. Reese. Molecular dynamics in arbitrary geometries: parallel evaluation of pair forces. Submitted to Molecular Simulation, 2007.

[3] J. G. Powles, S. Murad, and P. V. Ravi. A new model for permeable micropores. Chemical Physics Letters, 188:21-24, 1992.

[4] K.P. Travis, B.D. Todd, and D.J. Evans. Departure from Navier-Stokes hydrodynamics in confined liquids. Physical Review E, 55(4):4288-95, 1997.

[5] Herbert Goldstein. Classical Mechanics. Addison-Wesley, 2nd edition, 1980.

[6] D. C. Rapaport. The Art of Molecular Dynamics Simulation. Cambridge University Press, 2nd edition, 2004.

[7] M.P. Allen and D.J. Tildesley. Computer simulation of liquids. Oxford University Press, 1987.

[8] George Karypis and Vipin Kumar. METIS. A software package for partitioning unstructured graphs, partitioning meshes, and computing fill-reducing orderings of sparse matrices. Version 4.0. University of Minnesota, http://glaros.dtc.umn.edu/gkhome/views/metis.

[9] ParaView: Parallel Visualization Application. http://www.paraview.org.

[10] L. D. Landau and E. M. Lifshitz. Statistical Physics Part 1 (Course of Theoretical Physics v.5). Pergamon, 3rd edition, 1980.

[11] Thomas Werder, Jens H. Walther, and Petros Koumoutsakos. Hybrid atomistic-continuum method for the simulation of dense fluid flows. Journal of Computational Physics, 205(1):373-390, 2005.

[12] Charles Kittel. Introduction to solid state physics. Wiley, 8th edition, 2005. 